\title{
Choice based on exclusion in pigeons
}

\author{
TRICIA S. CLEMENT and THOMAS R. ZENTALL \\ University of Kentucky, Lexington, Kentucky
}

\begin{abstract}
When humans acquire a conditional discrimination and are given a novel-sample-comparison choice, they often reject a comparison known to be associated with a different sample and choose the alternative comparison by default (or by exclusion). In Experiment 1, we found that if, following matching training, we replaced both of the samples, acquisition took five times longer than if we replaced only one of the samples. Apparently, the opportunity to reject one of the comparisons facilitated the association of the other sample with the remaining comparison. In Experiment 2, we first trained pigeons to treat two samples differently (to associate Sample A with Comparison 1 and Sample B with Comparison 2) and then trained them to associate one of those samples with a new comparison (e.g., Sample A with Comparison 3) and to associate a novel sample (Sample C) with a different, new comparison (Comparison 4). When Sample B then replaced Sample C, the pigeons showed a significant tendency to choose Comparison 4 over Comparison 3. Thus, when given the opportunity, pigeons will choose by exclusion.
\end{abstract}

Humans are known to reason by exclusion (McIlvane et al., 1987; Meehan, 1995). For example, when humans are presented, in a conditional discrimination in which an initial or sample stimulus indicates which of two test or comparison stimuli is correct, with a novel sample and are given a choice between a familiar (defined) comparison already associated with a sample and a novel (undefined) comparison not yet associated with a sample, the novel comparison is typically chosen (by exclusion). Presumably, the participants eliminate from choice those comparisons that have learned associations with other samples. The inferred relationship between undefined comparisons and undefined samples has also been demonstrated in mentally retarded adults (Dixon, 1977) and in normal children (McIlvane, Munson, \& Stoddard, 1988).

Although, Tomonaga, Matsuzawa, Fujita, and Yamamoto (1991) found no evidence for the use of exclusion by chimpanzees, other research has suggested that chimpanzees can choose by exclusion (Beran \& Washburn, in press; Hashiya, \& Kojima, 2001; Tomonaga, 1993). Furthermore, both bottlenose dolphins (Herman, Richards, \& Wolz, 1984) and California sea lions (Kastak \& Schusterman, 2002; Schusterman \& Krieger, 1984) have been found to choose an unfamiliar object when presented with a novel sign (sample) in the presence of familiar alternatives.

To what extent is choice by exclusion in nonhumans a capacity unique to large-brained mammals, such as chimpanzees, dolphins, and seals? Research with pigeons has provided evidence that when they learn that

This research was supported by National Institute of Mental Health Grant MH-59194. Address correspondence to T. R. Zentall, Department of Psychology, University of Kentucky, Lexington, KY 40506-0044 (e-mail: zentall@pop.uky.edu). two different samples are associated with the same comparison, they represent those samples similarly, so that they can then be substituted for each other in other contexts (Urcuioli, Zentall, Jackson-Smith, \& Steirn, 1989; Wasserman, DeVolder, \& Coppage, 1992). The finding of such emergent stimulus relations suggests that pigeons are capable of forming classes made up of arbitrary stimuli. In other words, they are capable of class inclusion involving arbitrary stimuli. When similar behavior is demonstrated in humans, we say that the two stimuli have the same meaning. If pigeons can show class inclusion, can they also show class exclusion?

Early research suggested that pigeons do not choose by exclusion (Cumming \& Berryman, 1961). After pigeons had acquired an identity-matching task involving three colors (red, blue, and green), a novel color (yellow) was introduced. On trials involving the novel sample, novel comparison, and one of the familiar comparisons, the pigeons' choice of the matching comparison was near chance. However, pigeons have a natural tendency toward neophobia (i.e., they will not naturally choose a novel stimulus over a familiar one), which may have precluded positive results.

On the other hand, suggestive evidence that pigeons can be encouraged to learn a conditional discrimination by exclusion has been reported by Zentall, Edwards, Moore, and Hogan (1981). Pigeons were trained on a two-alternative conditional discrimination in which, for each sample, the incorrect comparison was consistent from trial to trial but the correct comparison varied among four different stimuli. When, on test trials, the correct comparison was replaced with a familiar stimulus, but one that was unfamiliar in the context of this sample, the pigeons chose it over the incorrect comparison. However, when the incorrect comparison was replaced (but the correct comparison remained), there was a substantial drop 
in choice accuracy. Thus, under these asymmetrical training conditions (multiple sample-correct-comparison associations), pigeons appear to learn to choose the correct comparison by excluding the incorrect one.

Further evidence that pigeons can be encouraged to learn a conditional discrimination by exclusion has been reported by Clement and Zentall (2000). Pigeons were trained on a conditional discrimination in which one sample (a yellow hue) was associated with one of two comparisons (vertical lines), and each of four other samples (red, green, blue, or white hues) was associated with the other comparison (horizontal lines). Evidence from retention tests (inserting a delay between the offset of the sample and the onset of the comparisons), as well as novel sample tests, indicated that the pigeons had learned the original task as if they were following the rule, "If the sample is yellow, choose vertical lines; otherwise, choose horizontal lines." That is, they had learned that yellow belonged to one class and, by exclusion, everything else belonged to the other.

In the present research, we asked whether pigeons would naturally respond as if they were using an exclusion rule to choose between comparisons without being induced to do so by asymmetrical training procedures (i.e., without making it difficult to learn the task unless they learned by exclusion). In two experiments, we asked whether a novel sample-comparison association would emerge if it was placed in the context of a familiar samplecomparison association.

\section{EXPERIMENT 1}

\section{Method}

\section{Subjects}

The subjects were 8 White Carneaux pigeons, purchased as retired breeders (5-8 years old) from the Palmetto Pigeon Plant (Sumter, SC). The pigeons were maintained at $80 \%$ of their freefeeding body weights throughout the experiment and were caged individually, with grit and water available in the home cage. The pigeons were maintained on a 12:12-h light:dark cycle. All the pigeons had previously served in an unrelated study involving simple simultaneous discriminations.

\footnotetext{
Apparatus

The experiment was conducted in a BRS/LVE (Laurel, MD) sound-attenuating pigeon test chamber. Three horizontally aligned rectangular response keys $(2.5 \mathrm{~cm}$ high $\times 3.0 \mathrm{~cm}$ wide $)$ were mounted on the response panel and were separated from each other by $1.0 \mathrm{~cm}$. Mounted behind each response key was a 12 -stimulus inline projector (Industrial Electronics Engineering, Series 10, Van Nuys, CA, with No. 1820 G.E. lamps) that projected red, green, blue, and white hues (Kodak Wratten Filters 26, 60, and 38a and no filter, respectively) and a white plus (13 $\mathrm{mm}$ long and $3 \mathrm{~mm}$ wide) on a black background. The left and right projectors could also project a white circle (with a $16-\mathrm{mm}$ outside and a $13-\mathrm{mm}$ inside diameter). A houselight located at the center of the chamber ceiling provided general illumination. A rear-mounted grain feeder was horizontally centered on the response panel midway between the pecking keys and the floor of the chamber. Reinforcement consisted of 1.5-sec access to Purina Pro Grains. White noise and an exhaust fan masked extraneous noise. The experiment was controlled by a microcomputer located in an adjoining room.
}

\section{Procedure}

At the beginning of each trial, a sample stimulus was presented on the center response key, and 10 pecks were required to turn it off and turn on the comparison stimuli on the left and right keys. One response to either comparison constituted a choice and ended comparison presentation. Comparison choices were followed by a 10 -sec intertrial interval and, if the response was correct, by reinforcement. Stimuli and their locations (left/right) were counterbalanced.

Pigeons were initially trained on a conditional discrimination with S1 (red) and S2 (green) samples mapped onto C1 and C2 comparison stimuli (red and green, counterbalanced). Each session consisted of 96 trials, 48 trials involving each sample. Each pigeon was trained to a criterion of 2 successive sessions at $90 \%$ or better accuracy on each sample type. Each pigeon was then overtrained for an additional 20 sessions.

The pigeons were then randomly assigned to one of two groups. For pigeons assigned to Group Single Novel, the transfer task consisted of one of the samples from Phase 1 (red or green) and a novel sample, with the same comparison stimuli as in initial training. Half of these pigeons experienced a novel white sample (Group Single Novel/S3), and the other half experienced a novel plus sample (Group Single Novel/S4). For pigeons assigned to Group Both Novel, the transfer task involved two new samples (white and plus, S3 and S4). Again, each 96-trial session consisted of 48 trials involving each sample type. The experimental design is presented in Table 1. To determine whether the pigeons would exclude one comparison and respond by default to the remaining comparison, the rate of acquisition of novel sample trials was compared for Group Both Novel and the two single novel groups.

\section{Results}

\section{Acquisition}

Pigeons acquired Phase 1 matching to criterion on an average of 8.0 sessions. Those pigeons that were subsequently assigned to one of the single novel groups took an average of 8.2 sessions to attain criterion, whereas those that were subsequently assigned to Group Both Novel took an average of 7.7 sessions.

\section{Test}

Pigeons assigned to Group Both Novel learned the white sample-comparison association to a criterion of $90 \%$ correct in a single session in an average of 15.8 sessions, and they acquired the plus sample-comparison association to that criterion in an average of 15.2 sessions. Pigeons assigned to Group Single Novel/S3 and Group

Table 1

Design of Experiment 1

\begin{tabular}{lll}
\hline \multicolumn{1}{c}{ Group } & \multicolumn{1}{c}{ Training } & \multicolumn{1}{c}{ Transfer } \\
\hline Single novel/S3 & red $\rightarrow$ red & red $\rightarrow$ red \\
& green $\rightarrow$ green & white $\rightarrow$ green* \\
Single novel/S4 & red $\rightarrow$ red & plus $\rightarrow$ red \\
& green $\rightarrow$ green & green $\rightarrow$ green \\
Both novel & red $\rightarrow$ red & plus $\rightarrow$ red \\
& green $\rightarrow$ green & white $\rightarrow$ green*
\end{tabular}

Note-Half of the pigeons were trained as above. The remaining pigeons were trained to choose the nonidentity comparison (not shown). For these pigeons, in transfer, the unchanged trial type also involved a nonidentity sample-comparison association. Acquisition of trial types with $*$ and with ${ }^{\dagger}$ represents the critical pairs to be compared. 
Single Novel/S4 learned the white sample-comparison association to criterion in an average of 2.0 and 3.5 sessions, respectively (see Figure 1). Because of large individual differences in the rate of acquisition for the pigeons in Group Both Novel, which resulted in a skewed distribution, the acquisition scores were transformed $(Y=$ square root of $X)$. The transformed acquisition scores were then averaged over the two trial types for Group Both Novel. A one-way analysis of variance (ANOVA) performed on the transformed data indicated that the two single novel groups acquired the transfer task significantly more quickly than did the both novel group $[F(1,6)=8.24, p<.05]$. If, more conservatively, instead of averaging the scores for the two trial types for Group Both Novel, one considers the lowest of the two scores (i.e., the sample-comparison association that was most rapidly acquired), the difference in the acquisition scores for the two groups is still significant $[F(1,6)=$ $7.17, p<.05]$. Thus, the pigeons in the single novel group appear to have used the familiar sample-comparison association as a basis for excluding one comparison when they were presented comparison stimuli following novel sample presentations.

Rapid acquisition by the single novel groups suggests that the pigeons in these groups may have shown positive transfer on the first test session. However, an examination of the first-session transfer data suggests that, on average, transfer was actually below chance for the single novel pigeons. The Session 1 transfer data are presented in Table 2. Furthermore, the first-session transfer data were highly variable for single novel pigeons. Two pigeons showed strong negative transfer, 1 showed strong positive transfer, and 1 responded at chance. Remarkably, despite the strong negative transfer (mean $=3.2 \%$ correct), these 2 pigeons attained the $90 \%$ correct acquisition criterion in an average of 3.5 sessions.

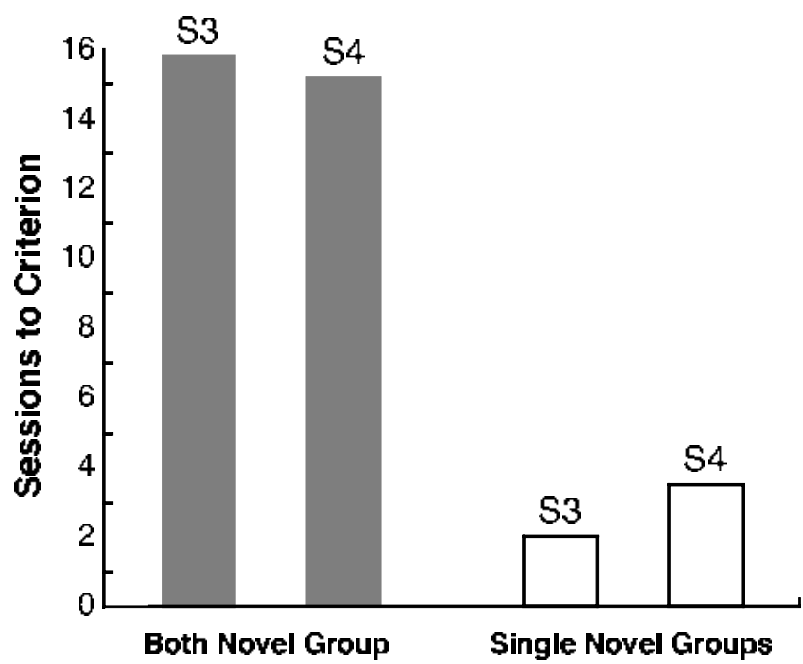

Figure 1. Experiment 1: mean sessions to criterion on novel sample trials (S3 and S4) for the pigeons in the both novel group and the pigeons in the two single novel groups.
Table 2

Experiment 1, Transfer Session 1: Percentage Correct on Novel Trial Types for Individual Subjects

\begin{tabular}{ccc}
\hline & Both Novel & Single Novel \\
\hline & 46.9 & 79.2 \\
50.0 & 2.1 \\
& 51.0 & 4.2 \\
$M$ & 45.8 & 50.0 \\
& 48.4 & 33.9 \\
\hline
\end{tabular}

Note-For pigeons in Group Both Novel, percentage correct has been averaged over the two novel trial types.

\section{Discussion}

When the pigeons that had acquired a matching task were required to learn a new matching task, acquisition was greatly facilitated when only one of the samplecomparison associations was new. Although one could argue that the pigeons that had to learn two new associations had more to learn than did those that had to learn only one new association, they also had twice as many learning trials. Furthermore, the pigeons that could not learn by exclusion took more than five times as many sessions, on average, to acquire their task ( 15.50 sessions) as those that could learn by exclusion ( 2.75 sessions).

Alternatively, the pigeons could have acquired the original matching task by learning to avoid the incorrect comparison-for example, if the sample was red, avoid the green comparison, and if the sample was green, avoid the red comparison. But such learning would not have provided a relevant cue when the novel sample was introduced. It is also possible, as one of the reviewers suggested, that during original training the pigeons learned to avoid the red comparison whenever the sample was not red (i.e., if the sample was not red, avoid the red comparison). However, any tendency to avoid the incorrect comparison would be considered a form of exclusion.

The fact that 2 of the pigeons in the single novel condition actually transferred well below chance, whereas 1 transferred well above chance, suggests that some subjects treated the novel white sample as red, whereas 1 subject treated it as green (or not red). It may be that, without some prior experience with the white hue, there is some ambiguity about how to treat the novel hue.

\section{EXPERIMENT 2}

A stronger case for the use of exclusion in a matching task can be made when immediate positive transfer is found following training. In Experiment 1, the introduction of novel sample stimuli that had to be discriminated from each other (Group Both Novel) or from the remaining familiar sample (Group Single Novel) may have delayed the appearance of group differences (some of the pigeons in the single novel condition actually transferred well below chance). In the second experiment, we pretrained the pigeons to differentiate between the two samples that would be used in the test. In Experiment 2, the pigeons were first given conditional discrimination 
training with two sample stimuli (S1 and S2) and two comparison stimuli (C1 and $\mathrm{C} 2$ ). The pigeons were then given training with one of those samples (S1) and a new sample (S3) and with a new pair of comparison stimuli (C3 and $\mathrm{C} 4)$. On a single test session, the pigeons were then given the $\mathrm{S} 1$ and $\mathrm{S} 2$ samples presented with the new, C3 and C4, comparison stimuli, and on S2-sample trials, reinforcement was provided nondifferentially for comparison choice. Thus, test session performance can be taken as a conservative estimate of transfer.

\section{Method}

\section{Subjects and Apparatus}

The subjects were 8 pigeons similar to those used in Experiment 1. The apparatus was the same as that used in Experiment 1.

\section{Procedure}

Phase 1: Pretraining. The pigeons were initially trained on a conditional discrimination with red and green samples mapped onto green and red comparison stimuli, respectively. In Experiment 2 , we decided to use nonmatching-to-sample during original training, to ensure that the results found in Experiment 1 could not in some way be attributed to the identity relation between the sample and the correct comparison. Following presentation of a red sample, choice of the green comparison was designated as correct, and following presentation of a green sample, choice of the red comparison was designated as correct. In all other respects, sessions, criterion performance, and overtraining were the same as those in Experiment 1. Each session consisted of 96 trials, with 48 trials of each sample type.

Phase 2: Training. During Phase 2, the pigeons received conditional discrimination training with one of the familiar (red or green) samples and a novel white sample, with new circle and plus comparisons. Sample-comparison associations were counterbalanced. Again, each session consisted of 96 trials, with 48 trials of each sample type. Following each session, 24 refresher trials involving Phase 1 trials were given, to ensure maintained familiarity with the previous training contingencies. Each pigeon was trained to a criterion of $90 \%$ correct or better accuracy for each of the sample types on a single session before advancing to the test phase.

Test phase. During a single test session, the pigeons received the red and green samples from Phase 1 with the circle and plus comparison stimuli from Phase 2. On trials involving the sample not previously trained with these comparisons, choice of either comparison was randomly reinforced on $50 \%$ of the trials. The design of Experiment 2 is presented in Table 3.

\section{Acquisition: Phase 1}

The pigeons acquired Phase 1 matching to criterion in an average of 15.2 sessions.

Table 3

Design of Experiment 2

\begin{tabular}{lll}
\hline Pretraining & \multicolumn{1}{c}{ Training } & Transfer Test \\
\hline $\begin{array}{l}\text { red } \rightarrow \text { green } \\
\text { green } \rightarrow \text { red }\end{array}$ & red $\rightarrow$ circle & red $\rightarrow$ circle \\
& white $\rightarrow$ plus & green $\rightarrow$ plus* \\
or & \\
red $\rightarrow$ green & white $\rightarrow$ circle & red $\rightarrow$ circle* \\
green $\rightarrow$ red & green $\rightarrow$ plus & green $\rightarrow$ plus \\
\hline
\end{tabular}

*Test trials (responding on these trials was nondifferentially reinforced).

\section{Acquisition: Phase 2}

The pigeons acquired the matching task involving one new sample and two new comparisons in an average of 24.0 sessions.

\section{Test}

The test data consisted of trials involving the sample from Phase 1 that had not been presented during Phase 2 . Initially, the data from the four separate counterbalancing groups were considered separately. A two-way ANOVA performed on the data from novel test trials, with the hue replaced in Phase 2 (red or green) and the shape associated with the replaced hue in Phase 2 (plus or circle) as factors, indicated that the main effects and the interaction were not significant. Thus, the data from novel test trials for the four counterbalancing groups were combined and were compared with chance (50\%).

The pigeons chose the comparison not previously associated with the other sample on an average of $71.9 \%$ of the test trials. A $t$ test performed on the data from the test session indicated that this difference was significantly above chance $[t(7)=4.93, p<.005]$. Every subject chose "by exclusion" at a level above chance on the test trials. The data from the novel test trials for the individual pigeons appear in Figure 2.

\section{Discussion}

The pigeons were first trained to distinguish between red and green samples and were then trained on a matching task with one familiar sample (either red or green) and a novel white sample associated with new comparisons. When the remaining sample from original training was substituted for the white sample, consistent with the results from the first experiment, the pigeons chose the comparison that was previously incorrect following the familiar sample on almost $72 \%$ of the test trials. Thus, the pigeons chose by excluding the comparison stimulus on the basis of sample-comparison associations already established, and they responded by default to the remaining comparison.

Choice by exclusion may also have occurred during Phase 2 of the experiment. Given that the red sample was already familiar and the white sample was not, the pigeons may have acquired the red $\rightarrow$ circle association first and then may have chosen the plus by default whenever the sample was not red. There is evidence that pigeons will adopt such a "strategy" when samples are used that consist of the presence versus the absence of a stimulus (e.g., a yellow hue vs. a dark key; Sherburne \& Zentall, 1993). That is, they appear to choose one comparison when a sample has been presented; otherwise they choose the other comparison. Perhaps they also choose by default when one of the samples is familiar but the other is not. If this is the case, in the test phase one should be able to replace the green sample with any novel sample (e.g., a blue hue) and obtain the same results. Whether the pigeons chose by exclusion in Phase 2 and then continued to do so when tested in the transfer 


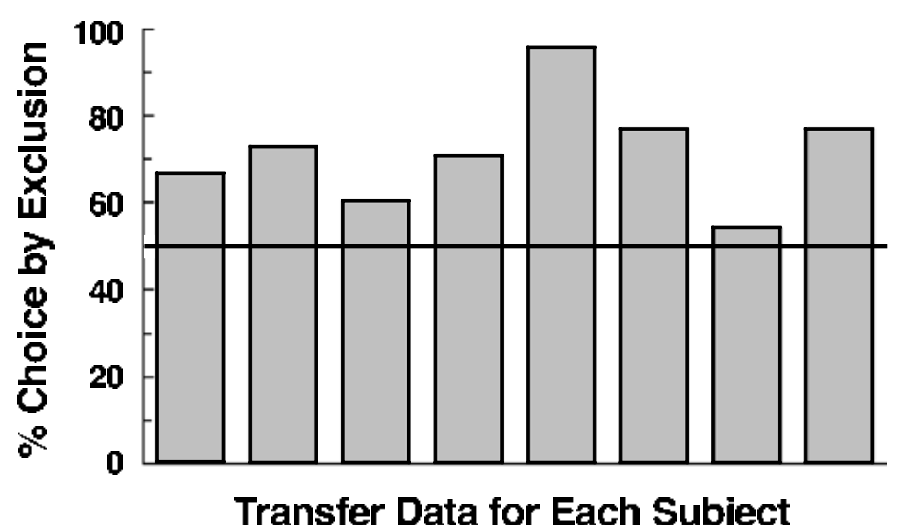

Figure 2. Experiment 2: percentages of choice by exclusion on the novel test trials for the individual pigeons.

phase or whether they began to choose by exclusion in the transfer phase, an emergent untrained relation was found that was indicative of rejection of the comparison associated with the alternative sample presented on other trials. In other words, it appears that the trained samplecomparison trials provided the context needed for choice by exclusion on untrained sample-comparison trials.

\section{GENERAL DISCUSSION}

The results of the two experiments are consistent in showing that pigeons can choose by means of exclusion. In both procedures, when confronted with unlearned sample-comparison associations, pigeons tended to choose a comparison by excluding the alternative. These results stand in contrast to the assumption that pigeons learn only stimulus-(correct)response relationships when exposed to conditional discriminations (Skinner, 1950). Furthermore, the fact that these and other instances of responding based on exclusion occur with nonhuman animals is inconsistent with the claim that such emergent processes require verbal behavior (Horne \& Lowe, 1996).

Although the present results suggest that pigeons can choose by exclusion, they do not explain how such learning develops, nor under what range of conditions it will occur. They do suggest that discrimination between samples may be necessary for exclusion to be found, because without such training, choice by exclusion does not seem to occur (see Session 1 transfer in Experiment 1). Furthermore, with such training (in Experiment 2), immediate choice by exclusion was found. Thus, the data suggest that in Experiment 2, it would not have been sufficient to replace the white sample with, for example, a novel blue sample.

Finally, although the present research suggests that pigeons can choose by exclusion, it is not clear whether this choice leads to new learning. For example, although pigeons show a reliable tendency to choose $\mathrm{C} 4$ in the presence of $\mathrm{S} 2$ because they reject $\mathrm{C} 3$, do they also learn the new S2-C4 association? When presented with S2, would they continue to choose $\mathrm{C} 4$ if, on other trials, the sample was novel (S4)? Data reported by Kastak and Schusterman (2002) suggest that, with practice, sea lions do acquire the novel associations that they originally choose by exclusion.

\section{REFERENCES}

Beran, M. J., \& WASHBurn, D. A. (in press). Chimpanzee responding during conditional matching-to-sample: Control by exclusion. Journal of the Experimental Analysis of Behavior.

Clement, T. S., \& Zentall, T. R.(2000). Development of a single-code/ default coding strategy in pigeons. Psychological Science, 11, 261264.

Cumming, W. W., \& Berryman, R. (1961). Some data on matching behavior in the pigeon. Journal of the Experimental Analysis of Behavior, 29, 565-601.

DiXoN, L. S. (1977). The nature of control by spoken words over visual stimulus selection. Journal of the Experimental Analysis of Behavior, 27, 433-442.

HashiYa, K., \& KoJIMA, S. (2001). Hearing and auditory-visual intermodal recognition in the chimpanzee. In T. Matsuzawa (Ed.), Primate origins of human cognition and behavior (pp. 155-189). Tokyo: Springer-Verlag.

Herman, L. M., Richards, D. G., \& Wolz, J. P. (1984). Comprehension of sentences by bottlenosed dolphins. Cognition, 16, 129-219.

HorNe, P. J., \& LOWE, C. F. (1996). On the origins of naming and other symbolic behavior. Journal of the Experimental Analysis of Behavior, 65, 185-241.

Kastak, C. R., \& Schusterman, R. J. (2002). Sea lions and equivalence: Expanding classes by exclusion. Journal of the Experimental Analysis of Behavior, 78, 449-465.

Mcilvane, W. J., Kledaras, J. B., Munson, L. C., King, K. A. J., DE Rose, J. C., \& STOdDARD, L. T. (1987). Controlling relations in conditional discrimination and matching by exclusion. Journal of the Experimental Analysis of Behavior, 48, 187-208.

Mcllvane, W. J., Munson, L. C., \& Stoddard, L. T. (1988). Some observations on control by spoken words in children's conditional discrimination and matching by exclusion. Journal of Experimental Child Psychology, 45, 472-495.

MeEhan, E. F. (1995). Emergence by exclusion. Psychological Record, 45, 133-154.

SCHUSTERMAN, R. J., \& KRIEGER, K. (1984). California sea lions are capable of semantic comprehension. Psychological Record, 34, 3-23. 
Sherburne, L. M., \& Zentall, T. R. (1993). Coding of feature and no-feature events by pigeons performing a delayed conditional discrimination. Animal Learning \& Behavior, 21, 92-100.

Skinner, B. F. (1950). Are theories of learning necessary? Psychological Review, 57, 193-216.

TomONAGA, M. (1993). Tests for control by exclusion and negative stimulus relations of arbitrary matching to sample in a "symmetry-emergent" chimpanzee. Journal of the Experimental Analysis of Behavior, 59, 215-229.

Tomonaga, M., Matsuzawa, T., Fujita, K., \& Yamamoto, J. (1991). Emergence of symmetry in a visual conditional discrimination by chimpanzees (Pan troglodytes). Psychological Reports, 68, 51-60.

Urcuioli, P. J., Zentall, T. R., Jackson-Smith, P., \& Steirn, J. N. (1989). Evidence for common coding in many-to-one matching: Re- tention, intertrial interference, and transfer. Journal of Experimental Psychology: Animal Behavior Processes, 15, 264-273.

Wasserman, E. A., De Volder, C. L., \& Coppage, D. J. (1992). Nonsimilarity-based conceptualization in pigeons via secondary or mediated generalization. Psychological Science, 3, 374-379.

Zentall, T. R., Edwards, C. A., Moore, B. S., \& Hogan, D. E. (1981). Identity: The basis for both matching and oddity learning. Journal of Experimental Psychology: Animal Behavior Processes, 7, 70-86.

(Manuscript received March 20, 2002; revision accepted for publication July 10, 2002.) 